\title{
Configurable 5G Air Interface for High Speed Scenario
}

\author{
Petri Luoto $^{\star}$, Kari Rikkinen ${ }^{\star}$, Pasi Kinnunen ${ }^{\dagger}$, Juha Karjalainen ${ }^{\dagger}$, Kari Pajukoski ${ }^{\dagger}$, \\ Jari Hulkkonen ${ }^{\dagger}$, Matti Latva-aho ${ }^{\star}$ \\ ${ }^{\star}$ Centre for Wireless Communications \\ University of Oulu, Finland \\ P.O. Box 4500, FI-90014 Oulu \{petri.luoto,kari.rikkinen, \\ matti.latva-aho\}@oulu.fi \\ ${ }^{\dagger}$ Nokia Bell Labs \\ Kaapelitie 4, \\ P.O. Box 319, FI-90620 Oulu \\ \{pasi.et.kinnunen,kari.pajukoski, \\ jari.hulkkonen\}@nokia-bell-labs.com
}

\begin{abstract}
In fifth generation (5G) networks one target is to provide very high capacity wireless access for the places where a lot of people consume a lot of data. Wireless communication is needed to provide access to high moving vehicles, however, extreme velocities must be taken into account in the design. Specific problems for air interface design to support extreme velocities are: high Doppler shifts, Inter-Carrier Interference (ICI), and difficulties in channel measurements needed for demodulation and hand-over measurements. Furthermore, very high data rates on outdoor macro cellular environment is challenging due to path loss. In high speed train (HST) deployments, the presence of lineof-sight connection enables the usage of wide bandwidths that are available on $\mathrm{cmWave}$ and mmWave spectrum. In this paper, we investigate the performance of mmWave single frequency network (SFN) in HST scenario. The performance of orthogonal frequency division multiplexing (OFDM) transmission with different new radio (NR) parameters is analyzed. Especially, the effect of Doppler and cyclic prefix (CP) is analyzed. Moreover, we conduct link level simulations and analyze the spectral efficiency in ideal HST scenario. Results show that it is possible to achieve very high data rates up to $10 \mathrm{Gbps}$.
\end{abstract}

Index Terms - cmW, eMBB, high speed train, mmW, new radio.

\section{INTRODUCTION}

The number of smart mobile devices is rapidly increasing and mobile Internet is expected to work everywhere, including high speed trains (HSTs). In a typical HST scenario, there is a big group of users in a train and they are consuming high data rate content, like e.g. enhanced Mobile Broadband (eMBB). High bandwidths, that are needed to provide very high capacity wireless access, are available only on cmWave and mmWave bands. In HST deployments, the presence of line-of-sight (LoS) connection enables to achieve macro cellular coverage on mmWave based communication. However, when trains move more than $350 \mathrm{~km} / \mathrm{h}$ existing wireless communications systems perform very poorly [1].

This research was supported by the Bittium Wireless Oy, Finnish Funding Agency for Technology and Innovation (TEKES), Keysight Technologies Finland Oy, Kyynel Oy, MediaTek Wireless Finland Oy, Nokia Solutions and Networks Oy, and University of Oulu under "High5" project (2192/31/2016).
The importance of HST communication has been recognized for years. Already in 2012, a novel Doppler frequency offset estimation method was proposed for the digital video broadcasting-terrestrial system in the HST environment [2]. They propose for using only a line-of-sight component for Doppler estimation is effective. In [3], Doppler shift is estimated through reconstructing the received signal based on discrete Fourier transform (DFT) at 2.6 GHz. In [4], [5], HST communication is looked from mmWave perspective together with large frequency bands.

However, more work is required in order to achieve $10 \mathrm{Gbps}$ connectivity to train that is moving $500 \mathrm{~km} / \mathrm{h}$. This creates a board field of challenges and research questions such as: How to compensate path loss when high carrier frequencies are used? How to couple the inter carrier interface (ICI) from high Doppler shifts that are 20 times more than in LTE? How to estimate and compensate a non line-of-sight Doppler? How to track the train accurately so that hand-overs are seamless?

In this paper, we focus on the analysis of the configurable air interface of new radio (NR) parameters for the high speed scenario. In this work, we analyze the impact of Doppler shift. Furthermore, we analyse the link budget and cyclic prefix (CP) length behaviour with different NR parameters. Finally, we show results from link-level simulations, especially block error rate (BLER) and spectral efficiency in the ideal scenario is analyzed. This paper is organized as follows. Doppler compensation is discussed is Section II. The system model is defined in Section III. Results from link budget, cyclic prefix, and link level simulation are analyzed in Section IV. Finally, Section V concludes the paper.

\section{DOPPLER COMPENSATION}

First, we analyze the impact of Doppler shifts in the achievable signal to interference plus noise ratio (SINR) of a received orthogonal frequency-division multiplexing (OFDM) signal, when no Doppler compensation is used. For the analysis, we assume that the channel between the transmitter and the receiver can be considered as a line-of-sight channel and 


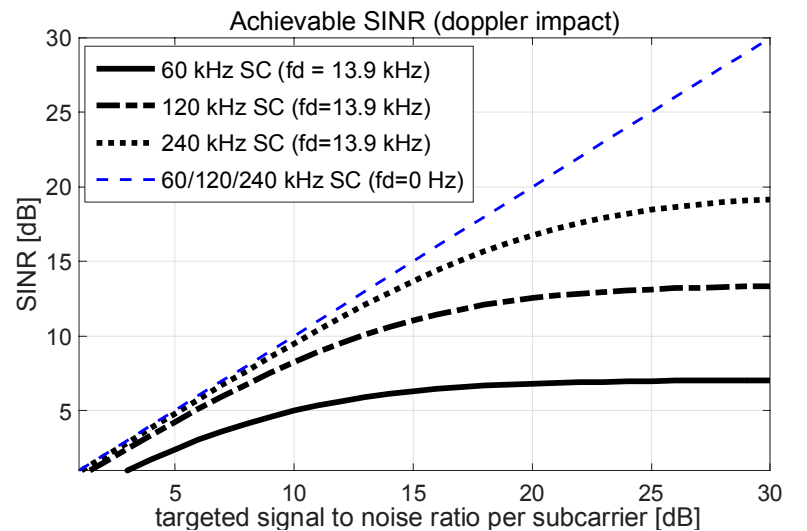

Fig. 1: Achievable SINR per subcarrier with different NR numerologies, when Doppler shift is $13.9 \mathrm{kHz}$.

the user equipment (UE), e.g., a transceiver located on top of a train, is moving with constant speed towards the base station. In this case, the achievable SINR per subcarrier can be expressed as

$$
\mathrm{SINR}=\frac{P_{s}}{P_{\mathrm{ICI}}+P_{\mathrm{N}}}
$$

where

$$
P_{s}=P \operatorname{sinc}^{2}\left(f_{d} T_{s}\right),
$$

is the useful signal power after the FFT, and

$$
P_{\mathrm{ICI}}=P\left(1-\operatorname{sinc}^{2}\left(f_{d} T_{s}\right)\right),
$$

is the ICI power caused by Doppler shift in the received OFDM signal [6], $P_{\mathrm{N}}$ is power of the thermal noise component, $P$ is transmit signal power per subcarrier, $f_{d}$ is the Doppler shift, $T_{s}$ is the OFDM symbol duration, and the channel gain is normalized to one.

Fig. 1 shows the achievable SINR as a function of targeted signal to noise ratio per subcarrier $\left(=P / P_{\mathrm{N}}\right)$ with different NR numerologies (OFDM subcarrier spacing $(\mathrm{ScS})$ values of $60 \mathrm{kHz}, 120 \mathrm{kHz}$, and $240 \mathrm{kHz}$, corresponding to OFDM symbol durations of $16.7 \mathrm{us}, 8.3 \mathrm{us}$, and $4.2 \mathrm{us}$, respectively). The carrier frequency is selected as $30 \mathrm{GHz}$, and the train speed is selected as $500 \mathrm{kmph}$, corresponding to a $f_{d}$ of 13.9 $\mathrm{kHz}$. In this case the achievable SINR is limited to $7.1 \mathrm{~dB}$, $13.4 \mathrm{~dB}$, and $19.6 \mathrm{~dB}$, for subcarrier spacing of $60 \mathrm{kHz}, 120$ $\mathrm{kHz}$, and $240 \mathrm{kHz}$, respectively. Thus, the results in Fig. 1 indicate clearly the need for Doppler estimation/compensation in high speed train scenario, especially when OFDM signals with low/moderate subcarrier spacing values, such as $60 \mathrm{kHz}$ or $120 \mathrm{kHz}$, are wanted to be deployed.

The Doppler shift in the received signal should be removed prior the receiver fast Fourier transform (FFT) processing to minimize the ICI. This can be implemented as part of the receiver automatic frequency control (AFC) functionality, as the Doppler shift due to LoS can be considered as constant (or very slowly varying) frequency shift in the received signal. Alternatively, the channel induced Doppler shift could be compensated already in the transmitter, provided that the

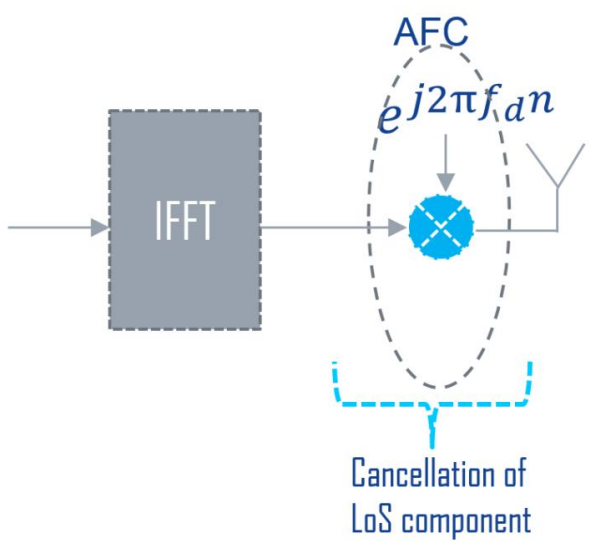

Fig. 2: Ideal Doppler compensation.

channel characteristics are known to the transmitter. In the HST scenario, the remote radio head (RRH) can estimate the Doppler shift from the received signal, and due to channel reciprocity, can utilize the information also for the transmission towards the train. Fig. 2 shows the functional model of the ideal Doppler compensation implemented in the transmitter side in RRH.

As the real propagation channel is not a perfect and fixed LoS channel, and neither the frequency error estimation is ideal, there occurs residual ICI component in the received signal after the FFT. For cancelling this component, additional frequency domain ICI cancellation could be deployed at the receiver after the FFT. In this work, we deploy cancellation of the LoS component assuming ideal estimation of the Doppler for the LoS path of the channel, but no further ICI cancellation after the FFT. The impact of Doppler estimation error and cancellation of NLoS components is left for further work.

\section{System Model}

A network with multi-input multiple-output (MIMO) with orthogonal frequency division multiplexing (OFDM) is considered. It consist of a set of remote radio heads (RRHs), each equipped with $N_{\mathrm{t}}$ transmit antennas (Tx) and a train equipped with $N_{\mathrm{r}}$ receive antennas (Rx).

In order to achieve tens of Gbps of data rate, a wide carrier bandwidth is needed, $N \times 100 \mathrm{MHz}$. Higher carrier frequencies can enable the leveraging of larger bandwidths. A high antenna gain is achieved by placing the antenna panels on the top of the train. Moreover, beamforming should be used in order to maintain the continuous high data rate. A dense deployment of remote radio heads is needed, with a bi-directional link to minimize the path loss between the train and RRHs. Finally, to make the handover smooth, a single frequency network (SFN) is needed. SFN transmission helps to mitigate the impact of rapid handover and problems with a train passing fast over the positions of RRHs. This scenario is defined in 3GPP [7], as shown in Fig. 3.

Bi-directional beamforming ${ }^{1}$ is considered where two an-

${ }^{1}$ In the link level simulations uni-directional beamforming is considered. 


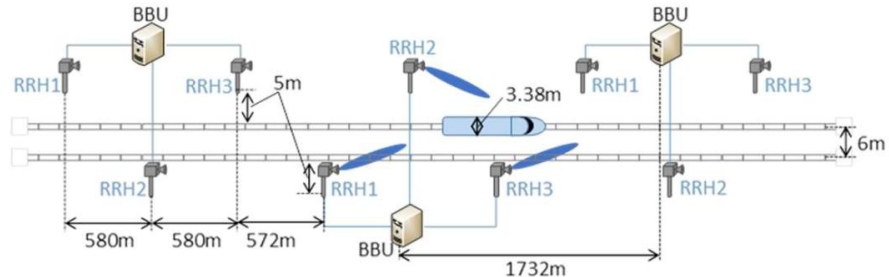

Fig. 3: High speed train scenario with remote radio heads and baseband units. [7]

TABLE I: Simulator parameters and assumptions.

\begin{tabular}{|l|l|}
\hline Parameter & Assumption \\
\hline Carrier frequency and coding & 30 GHz \\
\hline $\begin{array}{l}\text { Modulation } \\
\text { scheme } \\
\text { 256QAM 5/6 }\end{array}$ \\
\hline Channel coding & Turbo coding \\
\hline Available bandwidth & $1 \mathrm{GHz}$ \\
\hline FFT size & 2048 \\
\hline Subcarrier spacing & $60,120,240 \mathrm{kHz}$ \\
\hline Channel model & $\begin{array}{l}\text { CDL-D-13A (link level simula- } \\
\text { tions) }\end{array}$ \\
\hline K-factor & $13.3 \mathrm{~dB}$ and 7 dB \\
\hline Number of PRBs & 100 \\
\hline $\begin{array}{l}\text { Number of subcarriers per } \\
\text { PRB }\end{array}$ & 12 \\
\hline Antenna configuration & $128 \mathrm{Tx} \times 128 \mathrm{Rx}$ \\
\hline Number of layers & 2 \\
\hline Train speed & $500 \mathrm{~km} / \mathrm{h}$ \\
\hline Inter RRH distance & $580 \mathrm{~meters}$ \\
\hline Transmission power & $30 \mathrm{dBm}$ \\
\hline
\end{tabular}

tenna panels are directed to the opposite direction and the train receives/sends singals from/to multiple RRHs. The presence of the LoS component can be guaranteed as well as a spatial separation leading to a reduced RRH density.

\section{HIGH SPEED TRAIN NETWORK PERFORMANCE ANALYSIS}

Performance of results for OFDM radio links are presented in terms of SNR and block error rate (BLER). Furthermore, we show spectral efficiency when different modulation and coding schemes (MCSs) are used. Table I summarizes the main simulation parameters.

\section{A. Link budget analysis}

In the link budget analysis the following path loss models are used ${ }^{2}$

$$
\begin{aligned}
\mathrm{PL}_{\mathrm{dB}}= & 32.4+20 \log _{10}\left(d_{3 \mathrm{D}}\right)+20 \log _{10}\left(f_{c}\right) \\
\mathrm{PL}_{\mathrm{dB}}= & 32.4+20 \log _{10}\left(d_{3 \mathrm{D}}\right)+20 \log _{10}\left(f_{c}\right) \\
& -10 \log _{10}\left(\left(d_{\mathrm{BP}}\right)^{2}+\left(h_{\mathrm{BS}}-h_{\mathrm{UT}}\right)^{2}\right),
\end{aligned}
$$

where $d_{3 \mathrm{D}}$ is $3 \mathrm{D}$ distance in meters, $f_{c}$ is carrier frequency in $\mathrm{GHz}, d_{\mathrm{BP}}$ is break point distance, $h_{\mathrm{BS}}$ and $h_{\mathrm{UT}}$ are the effective antenna heights at the base station and the user terminal (train). The path loss model is given in [8], where it is described as

${ }^{2}$ See [8] how PL $1_{\mathrm{dB}}$ and PL $2_{\mathrm{dB}}$ are used based on the breakpoint distance.

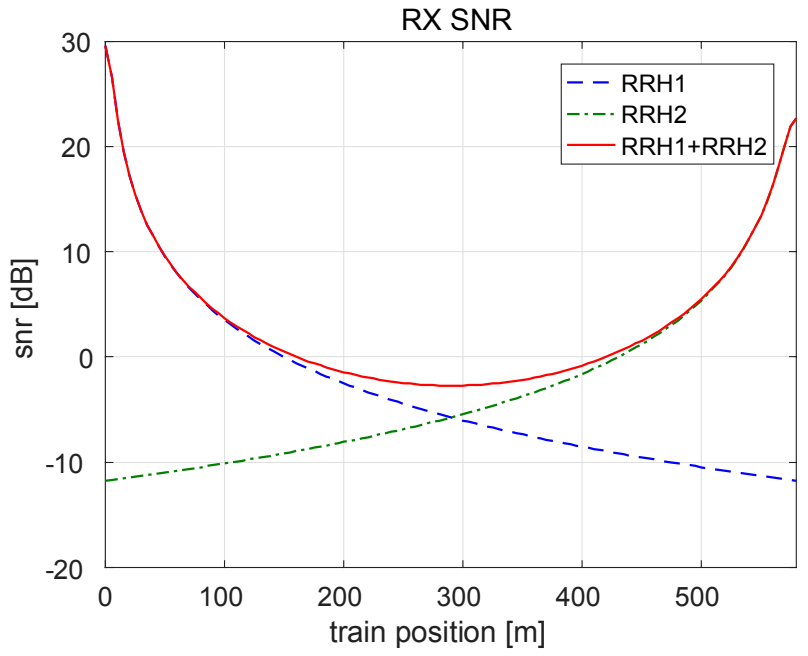

Fig. 4: Received SNR distribution between two RRHs.

urban macro LoS path-loss model. Used transmission power is $30 \mathrm{dBm}$.

In Fig. 4 we analyze SNR from the link budget. RRH1 is located in 0 meters and RRH2 is located in 580 meters, respectively). There is also two interfering RRHs (RRH0 located at -580 meters and RRH3 located at 1160 meters). When the signal is received only from RRH1 the minimum SNR would be around $-11 \mathrm{~dB}$ when the train is close to RRH2. This results shows that SFN network together with bi-directional beamforming should be considered, in order to achieve high enough SNR. When the signal is received from two RRHs the performance is lowest in the middle of RRHs, minimum received SNR is $-2.7 \mathrm{~dB}$. Bi-directional communication provides $8.3 \mathrm{~dB}$ gain over uni-directional communication.

\section{B. Cyclic prefix}

Cyclic prefix (CP) lengths follow NR parameters and it can expressed as follows:

$$
\mathrm{CP}_{\text {length }}=\frac{T_{\text {slot }}-N_{\text {symbols }} \frac{1}{\Delta_{\text {scs }}}}{N_{\text {symbols }}},
$$

where $T_{\text {slot }}$ is the slot duration $(0.125 \mathrm{~ms}$ for $60 \mathrm{kHz}$ or 120 $\mathrm{kHz} \mathrm{ScS}$ and $0.0625 \mathrm{~ms}$ for $240 \mathrm{kHz} \mathrm{ScS}), N_{\text {symbols }}$ defines the number of symbols $(6$ symbols for normal CP or 7 symbols extended $\mathrm{CP}$ when $60 \mathrm{kHz} \mathrm{ScS}$ is used, and 14 symbols for $120 \mathrm{kHz}$ or $240 \mathrm{kHz} \mathrm{ScS}$ ), and $\Delta_{\mathrm{ScS}}$ defines the subcarrier spacing.

Fig. 5 shows the impact of a CP length with different subcarrier spacings ( $\mathrm{ScSs}$ ). When the $\mathrm{CP}$ length is 1.2 us and $\mathrm{ScS}$ is $60 \mathrm{kHz}$, some performance loss around 100 meters can be seen (performance loss in 200 and 250 meters for ScS 120 $\mathrm{kHz}$ and $240 \mathrm{kHz}$, respectively). The reason is that RRH2 is outside CP window. Loss is relatively small due to the fact that case is ideal and environment is fully symmetric. Result reveal that it is beneficial to use long symbol duration, i.e., $\mathrm{ScS} 60 \mathrm{kHz}$. Moreover, if extended CP is used it increases the overhead and lowers the spectral efficiency. 


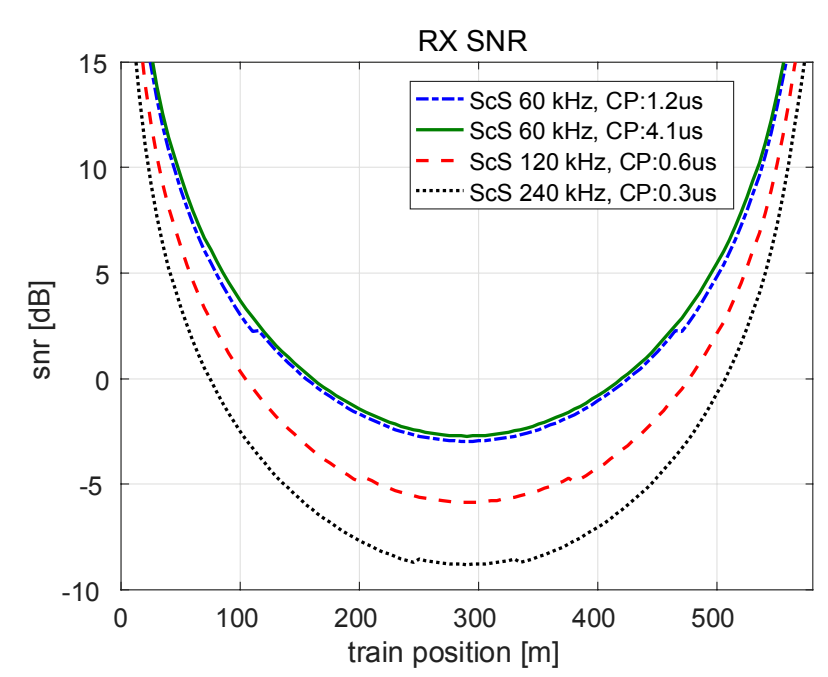

Fig. 5: Received SNR distribution with different CP lengths and different subcarrier spacings.

In Fig. 5, the reason for lower SNR with different ScSs is that the number of subcarriers is fixed, which results in 3 $\mathrm{dB}$ loss when $\mathrm{ScS}$ is doubled. However, from this result it is evident that extended $\mathrm{CP}$ would increase overhead leading to poor spectral efficiency. This means that RRH timing control is needed in order to avoid interference in SFN when bidirectional beamforming is used.

\section{Link level analysis}

In this analysis, 5G compliant link level simulator is used. We analyze BLER and spectral efficiency with different modulation and coding schemes (MSCs). We assume ideal Doppler estimation, i.e., Doppler shift is compensated $100 \%$ from the strongest channel tap. The used channel model in the simulations is CDL-D, with two K-factors ( $\mathrm{K}=7$ and $\mathrm{K}$ $=13.3$ ). With this channel model and high $\mathrm{K}$-factor, strong line-of-sight (LoS) channel is guaranteed as illustrated in Fig. 6. From this figure, we can see that there is roughly 45 $\mathrm{dB}$ difference between the strongest and the second strongest channel component when the train is located at 100 meters.

Fig. 7 shows the BLER vs. SNR curve when two MCSs are used, 64QAM with 3/4 code rate and 256QAM with 5/6 code rate. When 64QAM is used, performance in 50 meters and 100 meters is similar. Around $8 \mathrm{~dB}$ higher SNR is required if 256QAM is used. In the 256QAM case, a difference of 0.5 $\mathrm{dB}$ in the SNR can be seen when the location of the train changes from 50 to $100 \mathrm{~m}$. Fig. 8 shows the BLER vs. SNR curve when $\mathrm{K}$-factor $=7$. When compared with the previous result, the performance is $1 \mathrm{~dB}$ lower.

Finally, in Fig. 9 we show the spectrum efficiency as a function of SNR for different MCS sets when the train is in different positions. Results show that when the highest MCS is used, $10.7 \mathrm{bit} / \mathrm{s} / \mathrm{Hz}$ spectral efficiency is achieved. With 64QAM and 16QAM, the spectral efficiencies of $7.2 \mathrm{bit} / \mathrm{s} / \mathrm{Hz}$ and $3.2 \mathrm{bit} / \mathrm{s} / \mathrm{Hz}$ are achieved, respectively. When available

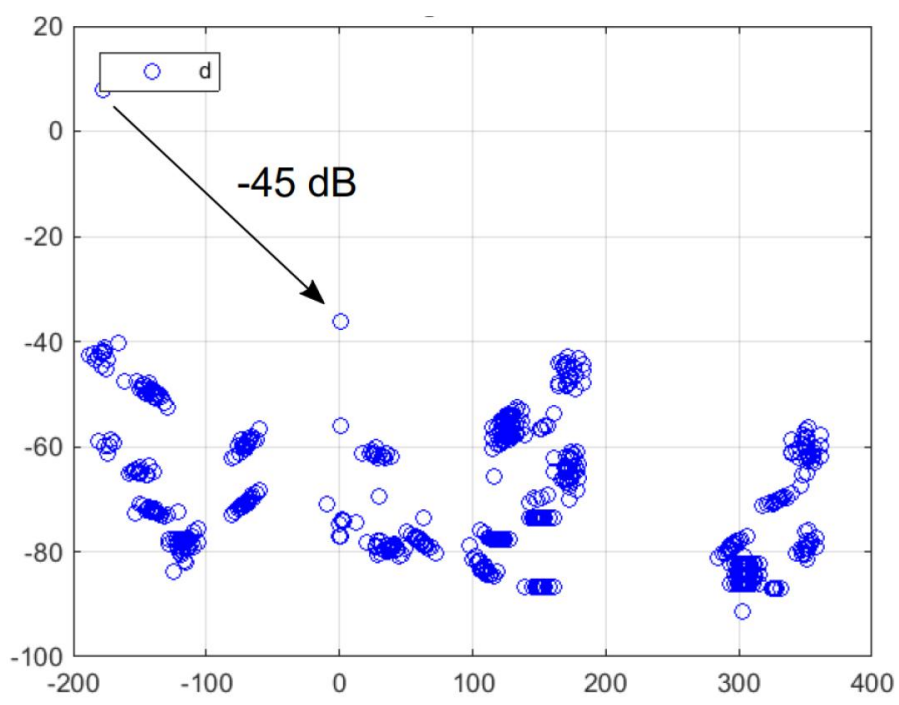

Fig. 6: Channel visualization when train is 100 meters away from RRH.

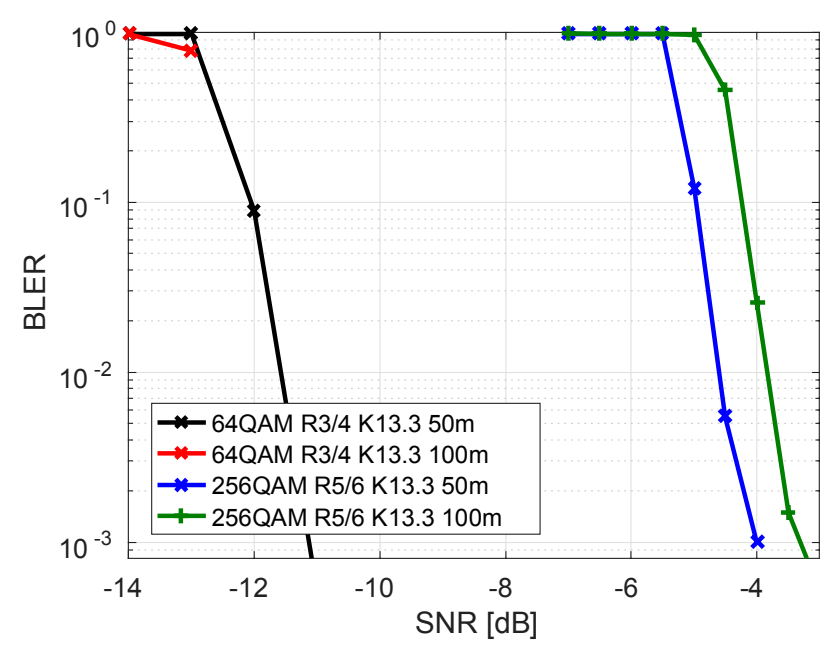

Fig. 7: BLER performance with 64QAM with coding rate $3 / 4$ and 256QAM with coding rate $5 / 6$, when $K=13.3$.

bandwidth is $1 \mathrm{GHz}$ it is possible to achieve data speed of 10 Gbps.

\section{CONCLUSION}

In this paper, we have investigated the performance of mmWave single frequency network (SFN) in HST scenario. We have analyzed the performance of orthogonal frequency division multiplexing (OFDM) transmission with different new radio (NR) parameters. We focussed on the effects of cyclic prefix and Doppler shift when different subcarrier spacings are used. Moreover, we analyzed the block error rate (BLER) and spectral efficiency using a link level simulator. Results revealed the utmost important timing correction in the remote radio heads (RRHs) when bi-directional beamforming in a single frequency network (SFN) is utilized. Although NR provides the opportunity to use flexible set of parameters, based on 


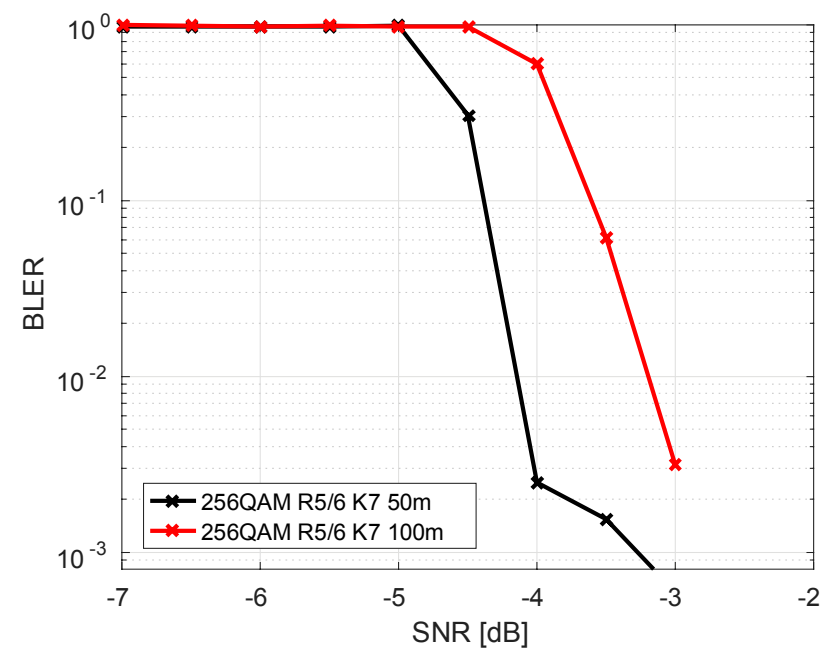

Fig. 8: BLER performance with 256QAM and coding rate 5/6, when $K=7$.

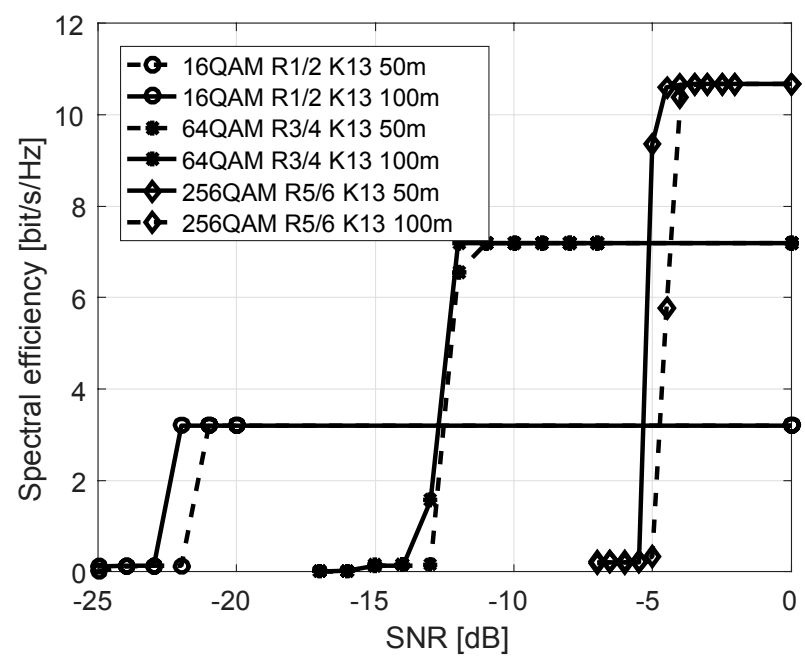

Fig. 9: Spectral efficiency vs. SNR for different MCSs and train positions. the results, long symbol duration is preferred, i.e., $60 \mathrm{kHz}$ subcarrier sparing. Moreover, results showed that it is possible to achieve a data rate of $10 \mathrm{Gbps}$. This paper is foundation for further studies. When Doppler estimate is accurate spectral efficiency is high. However, Doppler estimation errors require further studies. Moreover, in our future work, we will consider a more practical scenario and we will study the challenges of Doppler estimation when we have a train with two panels in SFN.

\section{REFERENCES}

[1] W. Luo, X. Fang, M. Cheng, and Y. Zhao, "Efficient Multiple-Group Multiple-Antenna (MGMA) Scheme for High-Speed Railway Viaducts,' IEEE Transactions on Vehicular Technology, vol. 62, no. 6, pp. 25582569, July 2013.

[2] L. Yang, G. Ren, and Z. Qiu, "A Novel Doppler Frequency Offset Estimation Method for DVB-T System in HST Environment," IEEE Transactions on Broadcasting, vol. 58, no. 1, pp. 139-143, March 2012.

[3] D. Fan, Z. Zhong, G. Wang, and F. Gao, "Doppler shift estimation for high-speed railway wireless communication systems with large-scale linear antennas," in 2015 International Workshop on High Mobility Wireless Communications (HMWC), Oct 2015, pp. 96-100.

[4] H. Song, X. Fang, and Y. Fang, "Millimeter-Wave Network Architectures for Future High-Speed Railway Communications: Challenges and Solutions," IEEE Wireless Communications, vol. 23, no. 6, pp. 114-122, December 2016

[5] Y. Cui, X. Fang, and L. Yan, "Hybrid Spatial Modulation Beamforming for mmWave Railway Communication Systems," IEEE Transactions on Vehicular Technology, vol. 65, no. 12, pp. 9597-9606, Dec 2016.

[6] S. Sesia, I. Toufik, and M. Baker, LTE, The UMTS Long Term Evolution: From Theory to Practice. Wiley Publishing, 2009.

[7] "3rd Generation Partnership Project, Technical Specification Group Radio Access Network, Study on Scenarios and Requirements for Next Generation Access Technologies, (Release 14), 3GPP TR 38.913 v14.2.0."

[8] "3GPP TR 38.900 V14.2.0. Study on channel model for frequency spectrum above $6 \mathrm{GHz}$ (Release 14)." 